\section{CLAUDIN-6 AFFECTS THE CELL CYCLE AND P53 SIGNALING IN GASTRIC CANCER}

Sanyog Dwivedi*. Cinvestav IPN, Mexico City, Mexico

Background Claudin-6 (CLDN6) differentially overexpressed in Gastric Cancer (GC) is associated with poor prognosis and survival of patients. Uncovering affected pathways and genes associated with CLDN6 in GC can help in the identification of novel prognostic targets.

Methods CBioPortal ${ }^{1}{ }^{2}$ was used to extract and analyze The Cancer Genome Atlas (TCGA) Stomach Adenocarcinoma PanCancer Atlas Data (STAD). FunRich tool ${ }^{3}$ and Gene Ontology molecular signature database of Gene Set Enrichment Analysis (GSEA) were used for functional enrichment. CBioPortal was used to identify differentially expressed genes between groups. Graph pad Prism 8 was used to generate the graphics.

Results TCGA STAD PanCancer Atlas data was analyzed in terms of alterations in CLDN6. 34\% of the GC samples (141 samples) were assigned to the CLDN6 alteration group while the rest of the samples (299) were assigned to the nonCLDN6 alteration group (figure 1). Major alterations of CLDN6 in GC included shallow deletion, diploid, gain, and differentially expressed mRNA (figure 2). Differentially overexpressed genes in the CLDN6 group with $\log$-ratio cutoff $\geq 1$ were used for functional enrichment in different biological pathway categories (figure 3); $18.1 \%$ of genes were associated with the transport of small molecules through the membrane, $14.6 \%$ mediated transmembrane transport and $12.5 \%$ were associated with lipid metabolism (figure 4). The Gene ontology molecular signature database of GSEA also confirmed that these genes were involved in lipid metabolism, transport activity, and epithelium development processes (table 1). Moreover, GC samples with CLDN6 alterations have higher mutations in p53 signaling (29\% in TP53, 7\% in CDKN2A) gene signature (figure 5) over samples in the non-CLDN6 alteration group (figure 6). Similarly, genes related with cell cycle control like CCNE1, MYC, SRC, and STAT3 showed higher mutations in the CLDN6 alteration group while JAK1 and E2F8 showed lower mutations than non-CLDN6 GC samples (figure 7). These observations indicate that CLDN6 in GC affects the transport of small molecules and lipid metabolism and that it is associated to tumors with higher mutations in p53 and cell cycle-related genes.

Abstract 14 Table 1 Functional enrichment of differentially expressed genes (log-ratio $\geq 1$ ) in CLDN6 group GC samples in Gene ontology molecular signature database of GSEA

\begin{tabular}{|l|l|l|l|}
\hline Gene Set Name [\# Genes (K)] & \# Genes in Overlap (k) & $\mathbf{p}$-value & FDR q-value \\
\hline GOBP EPITHELIUM DEVELOPMENT [1275] & 60 & $9.32 \mathrm{e}^{-21}$ & $9.49 \mathrm{e}^{-17}$ \\
\hline GOCC APICAL PLASMA MEMBRANE [351] & 31 & $1.33 \mathrm{e}^{-18}$ & $6.76 \mathrm{e}^{-15}$ \\
\hline GOCC APICAL PART OF CELL [414] & 33 & $2.25 \mathrm{e}^{-18}$ & $7.66 \mathrm{e}^{-15}$ \\
\hline GOCC CLUSTER OF ACTIN BASED CELL PROJE & 22 & $6.87 \mathrm{e}^{-18}$ & $1.75 \mathrm{e}^{-14}$ \\
\hline OJECTIONS [156] & & & \\
\hline GOMF TRANSPORTER ACTIVITY [1168] & 52 & $4.5 \mathrm{e}^{-17}$ & $9.16 \mathrm{e}^{-14}$ \\
\hline GOBP LIPID METABOLIC PROCESS [1426] & 57 & $1.49 \mathrm{e}^{-16}$ & $2.54 \mathrm{e}^{.13}$ \\
\hline GOCC BLOOD MICROPARTICLE [146] & 20 & $3.93 \mathrm{e}^{-16}$ & $5.72 \mathrm{e}^{-13}$ \\
\hline GOCC BRUSH BORDER [101] & 17 & $1.88 \mathrm{e}^{-15}$ & $2.4 \mathrm{e}^{-12}$ \\
\hline GOCC PLASMA MEMBRANE REGION [1208] & 50 & $3.32 \mathrm{e}^{-15}$ & $3.36 \mathrm{e}^{-12}$ \\
\hline GOBP EMBRYO DEVELOPMENT [992] & 45 & $3.43 \mathrm{e}^{-15}$ & $3.36 \mathrm{e}^{-12}$ \\
\hline
\end{tabular}

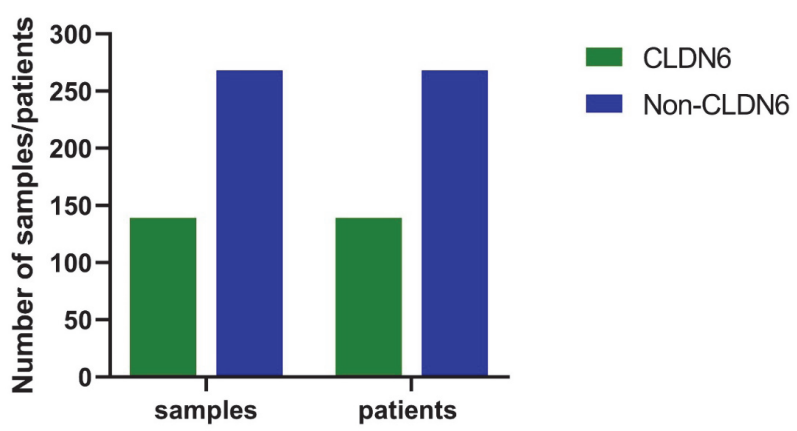

Abstract 14 Figure 1 Distribution of GC samples and patients of TCGA STAD data between CLDN6 and non CLDN6 alteration groups

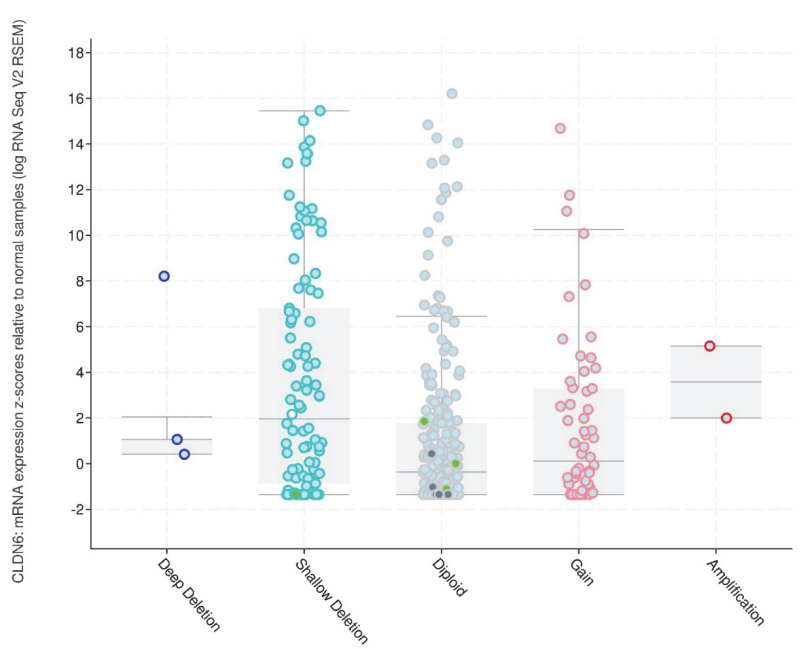

CLDN6: Putative copy-number alterations from GISTIC

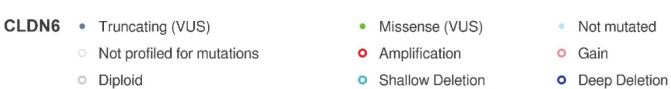

Abstract 14 Figure 2 Major alterations in CLDN6 in GC (TCGA STAD data)

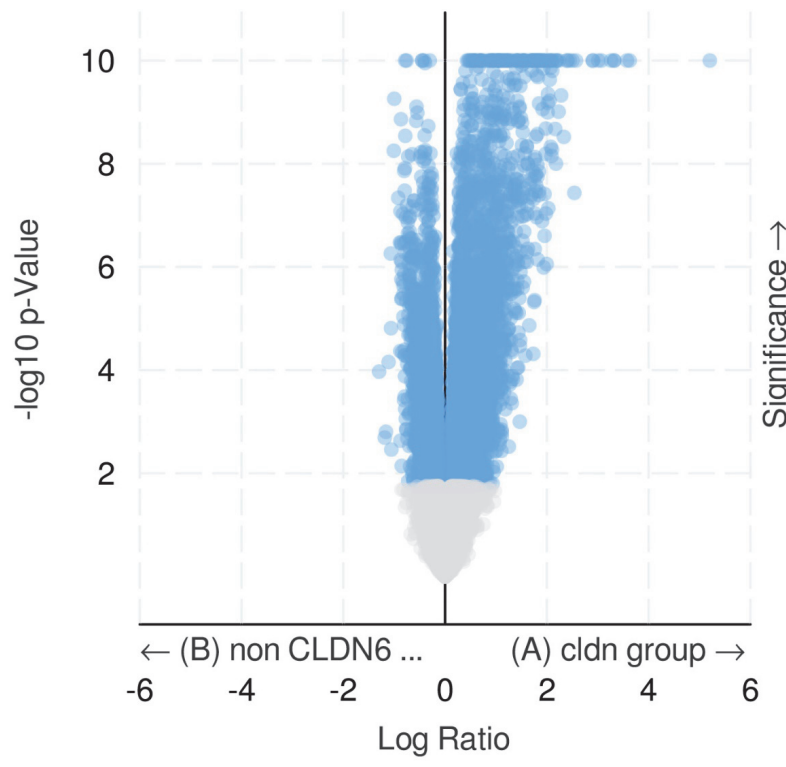

Abstract 14 Figure 3 Volcano plot of differentially expressed genes in CLDN6 and non CLDN6 groups. Log10 p-Value cutoff $\geq 2$ 


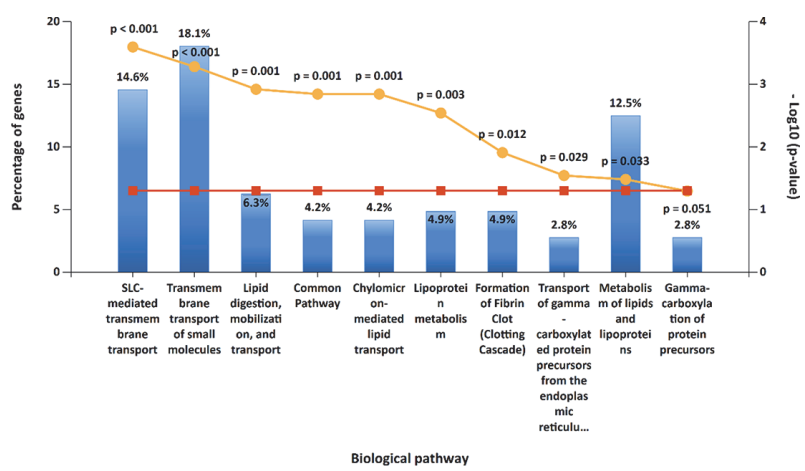

Abstract 14 Figure 4 Functional enrichment of differentially expressed genes (log-ratio $\geq 1$ ) in biological process category
Conclusions CLDN6 alterations in GC affect the cell cycle and p53 signaling pathways with higher mutations in TP53, CDKN2A, CCNE1, MYC, SRC, and STAT3 genes.

Acknowledgements This research was supported by CONACYT CVU grant 871712 .

\section{REFERENCES}

1. Cerami E, Gao J, Dogrusoz U, Gross BE, Sumer SO, Aksoy BA, et al. The cBio cancer genomics portal: an open platform for exploring multidimensional cancer genomics data. Cancer Discov 2012;2:401-4.

2. Gao J, Aksoy BA, Dogrusoz U, Dresdner G, Gross B, Sumer SO, et al. Integrative analysis of complex cancer genomics and clinical profiles using the cBioPortal. Sci Signal 2013:6:11.

3. Pathan M, Keerthikumar S, Ang C-S, Gangoda L, Quek CYJ, Williamson NA, et al. FunRich: An open access standalone functional enrichment and interaction network analysis tool. 2015PROTEOMICS;15:2597-601.

http://dx.doi.org/10.1136/jitc-2021-SITC2021.014

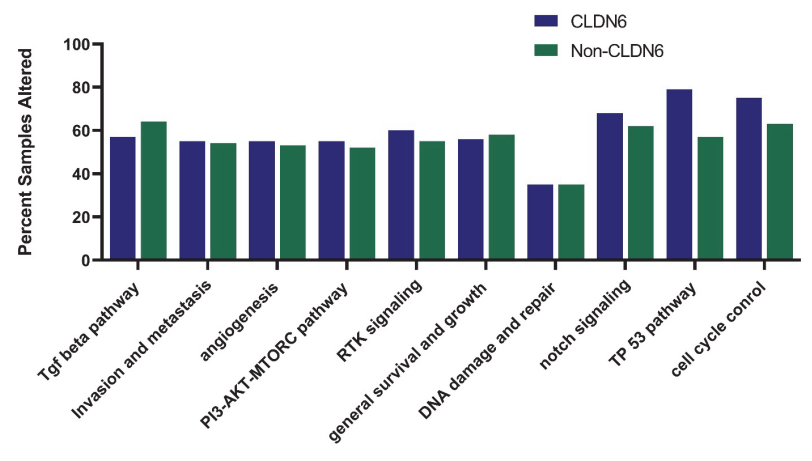

Abstract 14 Figure 5 Overall mutations in several important gene signatures involved in cancer between CLDN6 and non-CLDN6 GC samples

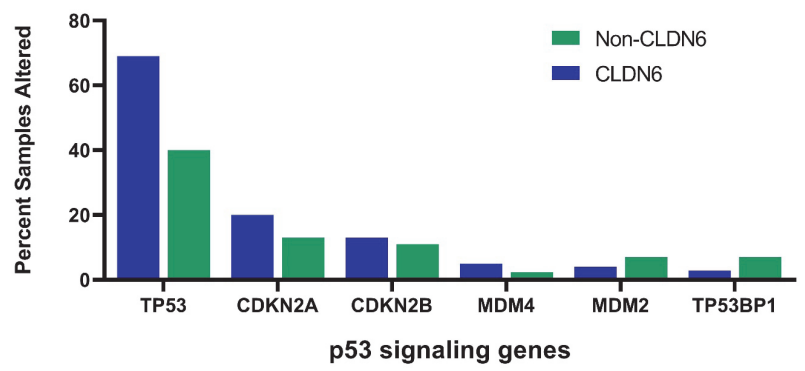

Abstract 14 Figure 6 Total mutations in p53 signaling genes

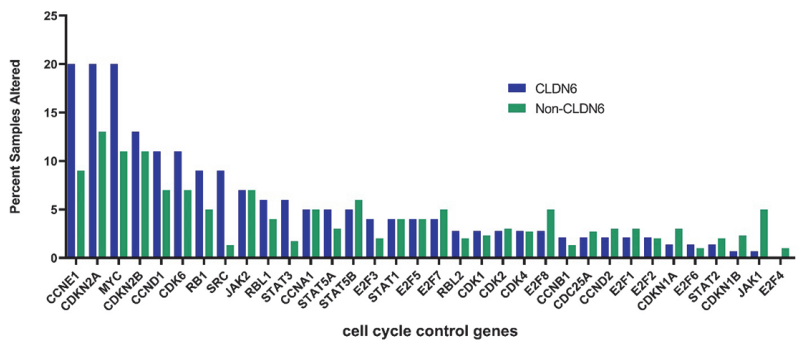

Abstract 14 Figure 7 Total mutations in genes involved in cell cycle control between CLDN6 and non-CLDN6 GC samples 